\title{
Working smarter by incorporating online testing: maximising self-management or opening an additional channel? A twelve month review.
}

\section{Kez Spelman, Taylor Hughes. Essex Sexual Health Service.}

Provide Community Interest Company was awarded a Lead Provider contract to deliver integrated sexual health services across the county of Essex from $1^{\text {st }}$ April 2016. Key to the model of service delivery was to maximize self management where safe and appropriate and therefore shift a proportion of face to face attendance to our new online self managed testing offer. A pivotal concern was that the availability of an online testing option would open a new channel for access and increase overall activity beyond what was affordable within the block contract, rather than shift activity from face to face attendance to online access. Using activity data from 2015-16 we established an activity projection which was compared to actual activity data from our online provision (SH24) and unified electronic clinical record (Inform).

Planned vs. Actual attendance during 2016-17

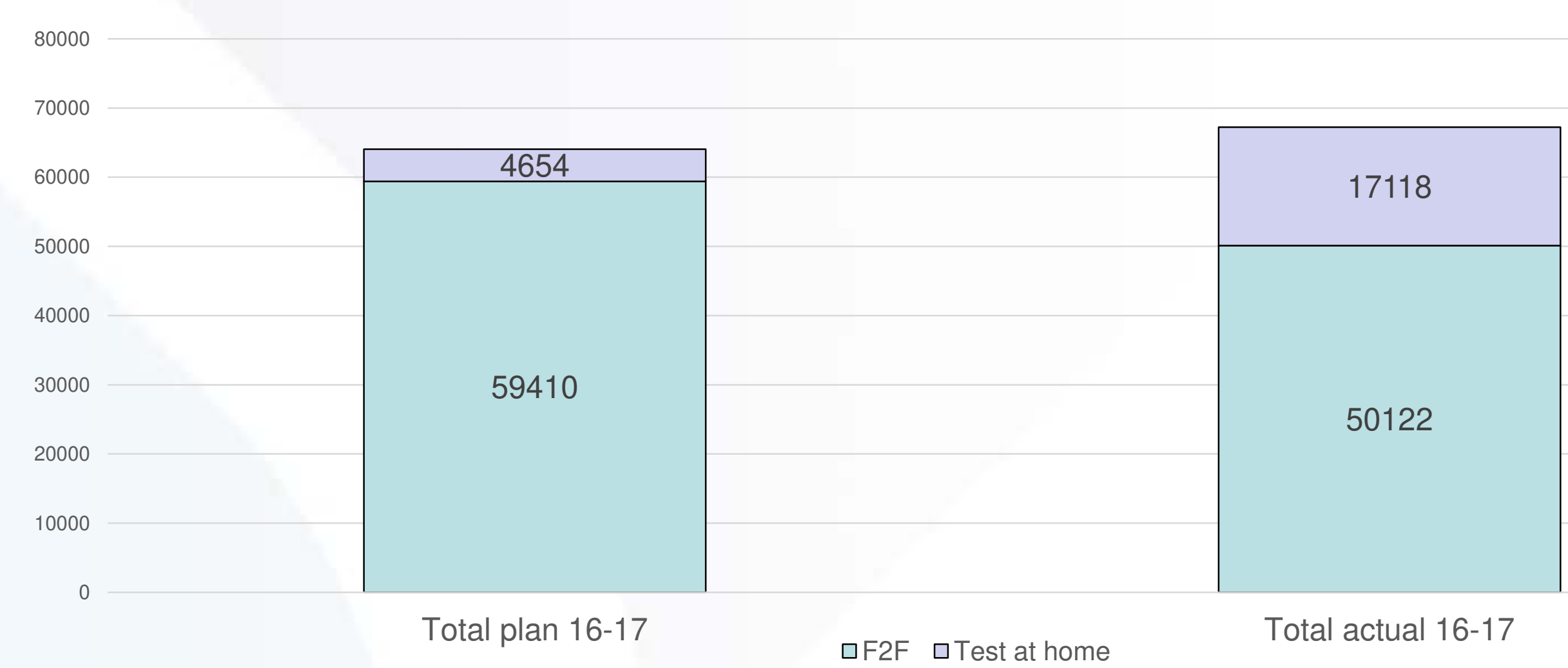

Within the year, face to face attendances fell by just over $9,000,(15 \%)$ whilst online testing uptake increased dramatically from plan to actuals. The overall increase in actual activity (both face to face and online) compared to plan was just over 3,000 (5\%). This indicates that although we have opened up a new channel of attendance for 3,000 people, we have reduced face to face attendance by 9,000 with associated saving of around $£ 400,000$.

Potential Shift

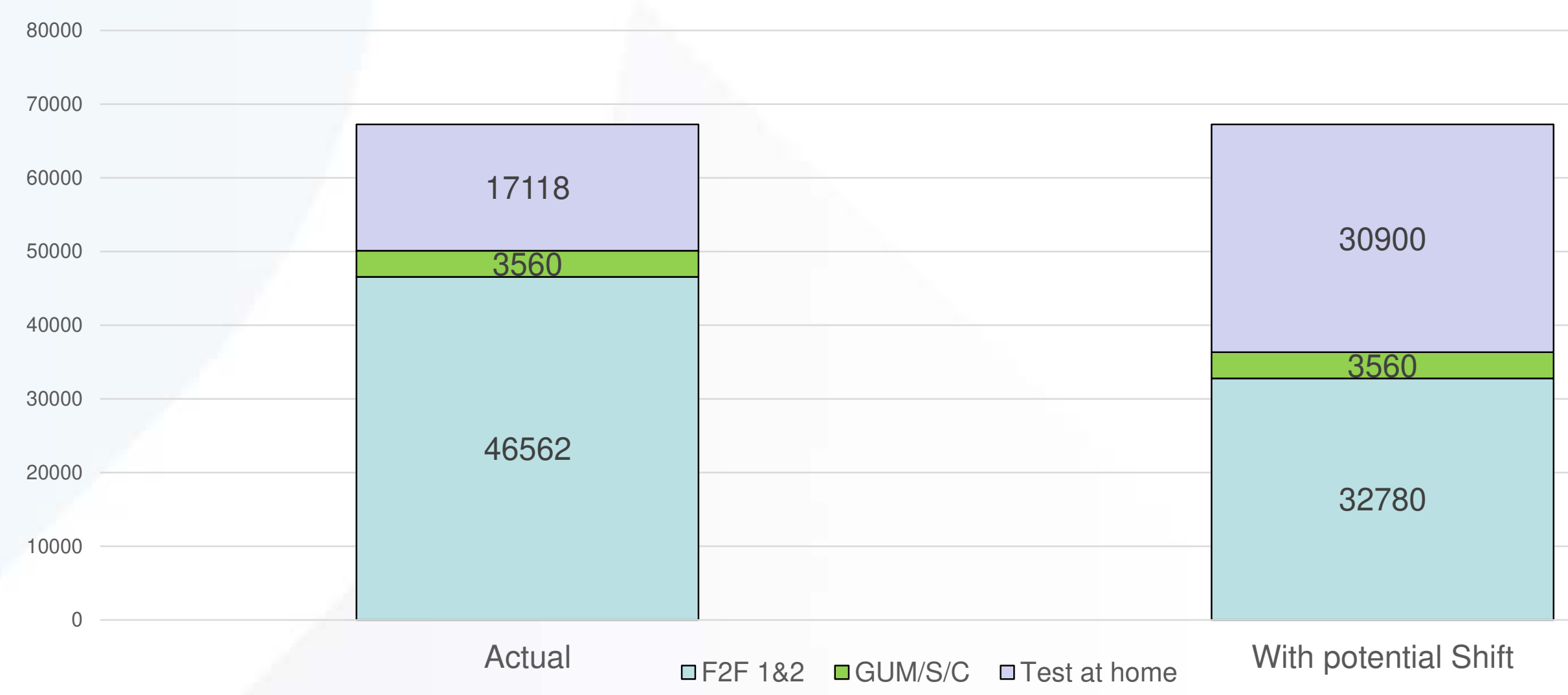

Further analysis of the face to face attendance data (by combining item of service, SHHAPT code and prescription) via the Inform record, enabled valid grouping of access type, to the following categories:

1. F2F level 1\&2, who could be seen by integrated sexual health nurses

2. Complex/Symptomatic (GUM/S/C), who need to be seen by a specialised clinician

3. Test at home, people who can safely and appropriately self-manage online

Conclusion

We can safely almost double our test at home numbers, and further reduce face to face attendance by around 14,000. This would equate to roughly a further $27 \%$ decrease in face to face attendance. This will be achieved through more effective triage and increased availability of online testing through our online booking system, which will help us ensure that people can maximize self-management of their care and release capacity in service for complex needs. 